Cipango
Cipango

15 | 2008

Guerre, colonialisme et commémoration

\title{
Les militaires coréens de l'armée japonaise et les procès pour crimes de guerre
}

Corean Soldiers in the Japanese Army and the War Crime Trials

\section{Tetsuya Takahashi}

Traducteur : Arnaud Nanta

\section{(2) OpenEdition \\ Journals}

\section{Édition électronique}

URL : https://journals.openedition.org/cipango/395

DOI : $10.4000 /$ cipango.395

ISSN : 2260-7706

\section{Éditeur}

INALCO

\section{Édition imprimée}

Date de publication : 1 janvier 2008

Pagination : 79-100

ISBN : 978-2-85831-177-4

ISSN : $1164-5857$

\section{Référence électronique}

Tetsuya Takahashi, «Les militaires coréens de l'armée japonaise et les procès pour crimes de guerre », Cipango [En ligne], 15 | 2008, mis en ligne le 11 novembre 2011, consulté le 30 juin 2021. URL : http:// journals.openedition.org/cipango/395; DOI : https://doi.org/10.4000/cipango.395

Ce document a été généré automatiquement le 30 juin 2021.

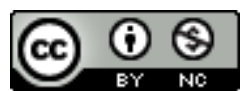

Cipango est mis à disposition selon les termes de la Licence Creative Commons Attribution - Pas d'Utilisation Commerciale 4.0 International. 


\title{
Les militaires coréens de l'armée japonaise et les procès pour crimes de guerre
}

\author{
Corean Soldiers in the Japanese Army and the War Crime Trials
}

\author{
Tetsuya Takahashi
}

Traduction : Arnaud Nanta
J'aimerais continuer à être de ce monde, fut-ce en tant qu'esprit. Et si c'est impossible, alors j'aimerais subsister dans le souvenir de quelqu'un. Testament de Cho Munsang 趙文相, 1947 .

Le sanctuaire Yasukuni, lieu de commémoration des hommes et des femmes tombés pour le Japon, célèbre quatorze criminels de guerre de catégorie A - traduits en justice pour crime contre la paix lors du Tribunal militaire international pour l'ExtrêmeOrient ou Procès de Tōkyō en 1946-1948 (sept furent exécutés, les sept autres sont morts en prison) - au sein de sa célébration commune (gōshi 合祀). Cette présence soulève un grand problème lors des visites qu'y effectue le Premier ministre japonais ${ }^{1}$. Nous souhaitons nous pencher ici non pas sur les criminels de guerre de catégorie A, catégorie qui désigne les responsables politiques et militaires, mais plutôt sur ceux des catégories $\mathrm{B}$ et $\mathrm{C}$, c'est-à-dire les personnes condamnées après la guerre respectivement pour « crimes de guerre » et pour « crimes contre l'Humanité ». Le sanctuaire Yasukuni célèbre en son sein 1054 personnes reconnues coupables et condamnées à mort après la défaite $\mathrm{du}$ Japon par des tribunaux pour les catégories $\mathrm{B}$ et $\mathrm{C}$, mis en place au Japon et ailleurs en Asie. Le discours tenu habituellement par le sanctuaire à propos des criminels de guerre (les catégories $\mathrm{A}, \mathrm{B}$ et $\mathrm{C}$, soit un total de 1068 personnes) consiste à souligner que :

Ces 1068 personnes qui ont perdu la vie de façon misérable ont servi de boucs émissaires lors d'un jugement unilatéral, de pure forme, que les forces alliées (États-Unis d'Amérique, Grande-Bretagne, Pays-Bas, Chine) ont prononcé contre le Japon. Nous, au sanctuaire Yasukuni, nous les appelons "martyrs de Shōwa» et nous les y célébrons au titre de divinités. ${ }^{2}$ 
On montrera dans cet article comment la "violence juridique » s'est abattue sur les criminels de guerre originaires des colonies, et, dans un premier temps, pourquoi leurs procès furent marqués par l'arbitraire. Sera ensuite analysée la façon dont ces tribunaux les ont considérés «en tant que Japonais » afin de légitimer leur action de justice. Le processus suivant lequel les condamnés à mort ont été enregistrés au sein du Yasukuni sera exposé dans un troisième temps, et nous terminerons par un examen de la façon dont les survivants furent déchus de leur nationalité japonaise.

\section{Par un simple hasard}

3 Le discours appelant "martyrs de Shōwa " les criminels de guerre condamnés à mort, et les considérant comme des boucs émissaires ou comme injustement condamnés, ou encore s'efforçant de restaurer leur honneur voire, à l'inverse, de glorifier leurs « hauts faits ", n'est pas spécifique au sanctuaire Yasukuni. On trouve un message tout à fait identique sur des stèles qui glorifient ces condamnés à mort dans leurs départements d'origine, telles la «Stèle aux valeureux guerriers martyrs de la patrie condamnés lors des procès de guerre» (Sensō saiban junkoku resshi no hi 戦争裁判殉国烈士の碑, 1957) du sanctuaire pour la Protection du pays (Gokoku jinja 護国神社) du département de Yamaguchi, la "Stèle aux vingt-deux valeureux martyrs » (Junkoku nijūni resshi no hi 殉国二十二烈士の碑，1969） du sanctuaire pour la Protection du pays du département de Ehime, ou encore la «Stèle de glorification et de commémoration des valeureux guerriers martyrs, exécutés par décision de justice " (Hōmushi junkoku resshi irei kenshō hi 法務死殉国烈士慰霊顕彰碑, 1984) du sanctuaire Kibitsu 吉備津神 社 dans le département d'Okayama. En outre, ce problème ne se limite pas au shintō, de tels exemples pouvant aussi être observés au sein de temples bouddhiques: par exemple la "Stèle funéraire pour les martyrs de Shōwa exécutés par décision de justice » (Shōwa junnansha hōmushi tsuitō hi 昭和殉難者法務死追悼碑), dressée aux côtés d'une "Salle des âmes héroïques » (Eireiden 英霊殿) dans le temple Kōyasan Oku no in 高野山奥の院. Autrement dit, on peut trouver dans un coin du Kōyasan, lieu saint de la secte ésotérique Shingon remontant au $\mathrm{IX}^{\mathrm{e}}$ siècle, classé patrimoine mondial par l'UNESCO en 2004, une stèle expliquant que les « criminels de guerre de catégorie A » et les "criminels de guerre de catégories B et C", soit un total de 1068 personnes, auraient tous été injustement condamnés et qu'il ne faudrait pas oublier les " hauts exploits » de ces individus qui auraient œuvré « dans le sens de la politique nationale » et "afin d'améliorer le sort du pays", stèle, qui plus est, dressée sur demande du "supérieur de la branche [du bouddhisme] Shingon de Kōyasan ».

De nombreux problèmes ont été soulignés au Japon à propos du Procès de Tōkyō et des procès des criminels de guerre de catégories $B$ et $C$, ce qui a eu pour effet d'en diffuser une vision négative. Il est vrai que ceux-ci ont été problématiques sur de nombreux points, comme on le montrera plus bas, mais on ne peut en conclure pour autant que l'ensemble de ces criminels de guerre doivent être blanchis, ni que les responsabilités des dirigeants durant la guerre ou celles concernant les crimes commis par l'armée japonaise sont effacées. Les problèmes touchant aux procès des criminels de guerre doivent être appréhendés avec précision, et surtout en se dégageant des discours du type de celui que véhicule le Yasukuni. On doit notamment souligner que le responsable suprême lors de la guerre, l'empereur Shōwa (Hirohito), fut exempté de tout jugement sur décision politique américaine; que des crimes de guerre de l'armée 
japonaise aussi graves que les activités de l'unité 731 et la guerre bactériologique ou encore le bombardement continu de Chongqing 重慶 de 1938 à $1943^{3}$ ne furent pas jugés ; que la responsabilité coloniale japonaise ne fut absolument pas soulevée ; que les crimes de guerre des pays alliés eux-mêmes et notamment les bombardements atomiques de Hiroshima et Nagasaki, ainsi que les bombardements sans discrimination de zones civiles ne furent absolument pas questionnés; ou encore que le choix des prévenus lors les procès des criminels de guerre de catégories $B$ et $C$ fut totalement arbitraire, des personnes qui auraient dû être jugées ne l'ayant pas été et d'autres qui n'auraient pas dû l'être l'ayant été; et donc que, en définitive, les personnes « injustement condamnées » ont effectivement été nombreuses et que les procédures suivies lors des procès furent extrêmement sommaires.

5 Les procès des criminels de guerre de catégories $B$ et $C$ furent organisés par sept pays les États-Unis, la Grande-Bretagne, les Pays-Bas, la France, l'Australie, la Chine et les Philippines - dans l'ensemble des territoires qui avaient connu des combats lors de la Guerre de l'Asie et du Pacifique. Au total, 5700 personnes furent jugées pour 2244 affaires, pour déboucher au final sur 934 condamnations à mort ${ }^{4}$. Ces procès ne furent pas tous iniques comme cela a été affirmé à l'époque au Japon de façon émotionnelle, toutefois, dans certains cas, l'arbitraire fut effectivement poussé à l'extrême. Voici un témoignage du procureur australien John Williams, qui jugea près de cent personnes en trois mois à propos d'exactions commises sur des prisonniers de guerre détenus par l'armée japonaise.

Les ex-prisonniers qui se sont présentés à la quasi-totalité des procès de criminels de guerre ne connaissaient même pas le nom des Japonais inculpés et ne pouvaient les identifier que par des surnoms ou par des appellations familières. Il était donc impossible d'avoir des preuves décisives contre les criminels de guerre. Tous les gens qui ont été reconnus coupables de crimes de guerre ont simplement été jugés par hasard. On pourra penser que ces procès n'en avaient que le nom, tant ils étaient mal organisés, mais pouvait-on faire autre chose que mettre en place ces tribunaux? On ne pouvait résoudre ces problèmes d'aucune autre façon. À l'époque, on avait une grande masse de militaires japonais qui s'étaient rendus [aux troupes alliées] dans l'ensemble de l'Asie du Sud-Est. Aurait-on dû les laisser partir comme cela sans rien faire? Aurait-on dû les laisser se faire assassiner au coin des rues par les militaires hollandais ou par les Chinois et les Singapouriens présents ?5

Dans tous les cas de figure, les procès des criminels de guerre constituent un stéréotype de ce que Walter Benjamin appelle la « violence mythique " (die mytische Gewalt). Il ne s'agit pas, au travers de l'acte de jugement, d'annuler une frontière, mais bien plutôt d'en fixer une, qui sert à distinguer entre vainqueur et vaincu tout en posant les caractéristiques de la « violence fondatrice de droit » (die rechtsetzende Gewalt) qui posa la «Loi » au fondement de l'ordre mondial consécutif à la Seconde Guerre mondiale. Selon Benjamin, il s'agit d'une violence «imposant tout ensemble la faute et l'expiation », une violence "qui menace », une violence "sanglante » et qui « exige le sacrifice ». Le cas des procès de criminels de catégories $B$ et $C$ lors desquels l'arbitraire fut poussé à l'extrême comporte parfaitement ce type de caractéristiques. Quand il n'y a pas de preuve décisive permettant de savoir qui est réellement coupable, et que des gens « ont simplement été jugés par hasard $»^{7}$, le verdict ordonnant la peine de mort apparaît comme une violence qui «vient de la sphère incertaine et ambiguë du destin $»^{8}$, comme la violence qui toucha Niobé dans la mythologie grecque. 


\section{Les Coréens en tant que Japonais}

7 Parmi les personnes qui furent jugées lors des procès des catégories $B$ et $C$, figuraient également des criminels de guerre originaires des colonies, à commencer par 173 Taïwanais et 148 Coréens, des personnes des îles de Saipan et de Rota ou encore des ethnies Orok (Ulta) et Nivkhi (Giliak) de Sakhaline. Parmi ceux-ci, vingt-trois Coréens et vingt-et-un Taïwanais furent condamnés à mort, et sont actuellement commémorés au sein de la célébration commune du sanctuaire Yasukuni au titre de «martyrs de Shōwa ». Comme nous l'avons dit, il existe au Kōyasan Oku no in une "Stèle funéraire pour les martyrs de Shōwa exécutés par décision de justice " sur laquelle sont gravés, classés par région d'origine, les noms des criminels de guerre de catégories $\mathrm{A}, \mathrm{B}$ et $\mathrm{C}$ condamnés à mort : sur cette pierre figurent également les noms des soldats originaires de Corée et de Taïwan. Parmi eux, on remarque notamment Hong Sa'ik 洪思翊 $(1889-1946)^{9}$, un Coréen qui était général de corps d'armée dans l'Armée de terre japonaise et commandait un camp de prisonniers aux Philippines; cependant, en réalité, les hauts gradés étaient peu nombreux parmi eux et la majorité de ces Coréens remplissaient des fonctions de traducteurs ou de gardiens dans les camps de prisonniers, ou bien d'auxiliaires militaires. Ces derniers, les auxiliaires, étaient considérés comme inférieurs aux soldats japonais et devaient une obéissance absolue à leurs supérieurs ${ }^{10}$. Nous allons nous concentrer ci-après sur le cas de l'auxiliaire militaire Cho Munsang, dont le nom figure sur cette pierre commémorative.

Cho Munsang 趙文相 (1921-1947) est né dans la ville de Kaesòng 開城, en Corée ${ }^{11}$, dans une pieuse famille chrétienne. Alors qu'il étudiait à l'Université impériale de Keijō (Seoul; Keijō teikoku daigaku 京城帝國大學, fondée en 1926), il s'engagea comme gardien de prisonniers, répondant à un appel à recrutement ; il travailla ensuite comme gardien et traducteur dans un camp situé à proximité de Thanbyuzayat, en Birmanie (actuel Myanmar). Sa tâche consistait à envoyer, sur demande de ses supérieurs, des prisonniers anglais et australiens sur les chantiers de construction de la Ligne Thaïlande - Birmanie (la célèbre ligne «Taimen tetsudō» 泰緬鐵道, appelée « Death railway " par les Anglo-australiens) que l'armée japonaise construisit en 1942. Les Coréens recrutés comme auxiliaires militaires et qui servaient comme gardiens dans ces camps de prisonniers, étaient situés aux extrémités du système, pourtant c'est directement contre eux que se portait la haine que les militaires des forces alliées vouaient à l'encontre de l'armée japonaise, du fait des traitements inhumains et du travail forcé qu'ils leur faisaient subir. Lors de son procès, l'accusation contre Cho Munsang se concentra sur sa participation au lynchage d'un prisonnier américain dénommé Zunmo: Cho rejeta fermement cette accusation et affirma, «en jurant sur Dieu ", ne pas avoir participé à cet acte. Lorsque le procureur apprit que Cho était chrétien, il abandonna cette accusation et l'inculpa d'une autre charge, le passage à tabac de prisonniers.

Le procureur: "Tu veux nous dire que tu as été fidèle aux ordres de tes supérieurs, mais que tu n'as pas été fidèle à l'enseignement de la Bible ni à ta conscience?»

Cho: "A l'armée, la conscience personnelle ou la compassion religieuse n'ont aucune importance."

Le procureur : "Et est-ce que tu n'as jamais traité des prisonniers avec violence?"

Cho: "Je reconnais que j'en ai tabassé certains, oui, cela je l'ai fait."

Le procureur : "La cruauté ne figure pas dans les enseignements du christianisme! »

Cho: "Cela dépend de la façon dont on interprète la notion de cruauté. Ces tabassages,

c'était une attitude tout à fait quotidienne de l'armée japonaise. Mais si vous considérez que 
le tabassage relève de la cruauté, alors peut-être que j'ai effectivement été cruel. Lorsque j'étais au centre d'entraînement [militaire] de Pusan [en Corée japonaise], on m'a appris que les prisonniers devaient être traités comme des animaux. $\|^{12}$ pour avoir passé à tabac des prisonniers. Il fut pendu le 25 février 1947 dans la prison de Changi à Singapour, achevant sa vie au jeune âge de 26 ans. Dans son testament, on trouve le passage suivant, rédigé la veille de sa mort: «Kaneko ${ }^{13}$ chante d'une voix languissante: «Dans l'Autre Monde, j'espère qu'il n'y aura plus de distinctions telles que Japonais ou Coréens ». Pourquoi faut-il s'opposer et se haïr dans ce monde mouvant où nous n'existons qu'un vain instant? Les Japonais, les Coréens, tout cela n'existe pas. N'est-on pas tous des Asiatiques? Et d'ailleurs, c'est pareil pour les Occidentaux. Ah! demain j'irai me présenter le cœur joyeux ». Cho se demandait pourquoi il fallait « s'opposer et se haïr » entre «Japonais » et «Coréens ». Nous ne pouvons pas ne pas voir ici planer, dans ces paroles, l'ombre de la domination coloniale et de son immense violence, dont la vie et la mort de Cho Munsang furent les jouets. Arrêtons-nous un instant à ce sujet sur le passage suivant de son testament :

J'ai eu une vie bien mouvementée. J'ai passé ces vingt-six années comme dans un rêve. Quelle meilleure expression que "Juste une étincelle sur un caillou "? J'ai complètement oublié qui j'étais et ce que j'ai fait durant cette courte vie. Juste du mensonge et des singeries... Pourquoi n'ai-je pas vécu juste encore un peu plus? Tout ce que j'aurais dû faire et vivre par moi-même, quand bien même j'eus été malheureux et idiot, que cela soit pour mes connaissances ou pour ma façon de penser, tout cela, je l'ai emprunté à d'autres. Et le plus désespérant est que j'ai même cru que tout cela était à moi. Mon ami ! mon frère! possède par toi-même tes propres connaissances et ta propre pensée. Maintenant que la mort me fait face, je suis stupéfait de constater que je n'ai presque rien qui soit à moi. ${ }^{14}$

Cho Munsang possédait aussi le nom japonais Hirahara Moritsune 平原守矩. Il était «sujet» de l'Empire du Grand Japon depuis sa naissance et était «Japonais » de nationalité, tout en étant cependant "Coréen» en ce qu'il relevait des «territoires extérieurs » à la métropole (gaichi 外地) et ne pouvait pas entrer dans le cadre de la Loi sur l'état civil (Koseki hō 戸籍法); il était de ce fait l'objet de ségrégations diverses. Tout en voyant nié son caractère national ou ethnique par les politiques d'assimilation - appelées à l'époque "impérialisation ${ }^{15}-$, il faisait en même temps l'objet d'une ségrégation du fait de cette violence juridique qu'était la Loi sur l'état civil. De plus, la domination coloniale elle-même n'avait été rendue possible que grâce à cette " violence fondatrice de droit »: celle des traités que le Japon imposa à la Corée sur fond de pressions militaires depuis la fin du xix siècle jusqu'au Traité relatif à l'annexion de la Corée, de 1910. Cette violence légale s'est ensuite transformée en système en tant que " violence conservatrice du droit » (die rechtserhaltende Gewalt) ${ }^{16}$, avec la mise en place du Gouvernement général de Corée (Chōsen sōtokufu 朝鮮総督府), ainsi que de la police et des forces de gendarmerie (Kempeitai) ${ }^{17}$.

11 En outre, quand on réfléchit au contexte dans lequel Cho Munsang fut envoyé comme gardien dans un camp de prisonniers en Asie du Sud-Est, on ne peut que souligner que le Décret sur la Conscription (Chōhei rei 徽兵令) du mois d'août 1943, appliqué à l'ensemble du territoire coréen d'après la Loi réformant le Service militaire de mars 1943 (Kaisei heieki hō 改正兵役法), constituait une nouvelle forme de violence légale. Il est vrai que les gardiens coréens dans les camps de prisonniers n'étaient pas des militaires stricto sensu, mais des auxiliaires militaires, car ils y étaient en poste suite à un recrutement mené dans l'ensemble de la Corée en mai 1942, c'est-à-dire avant l'entrée en vigueur du Décret sur la Conscription. Cependant, le gouvernement japonais 
avait déjà pris diverses mesures suite à l'élargissement de la seconde Guerre sinojaponaise, initiée en 1937, notamment l'introduction du système militaire sur la base du volontariat pour les Coréens en février 1938 afin de mobiliser ceux-ci dans l'effort militaire. À l'entrée dans la Guerre du Pacifique, s'appuyant sur une analyse selon laquelle : «Ayant à l'esprit la difficulté de maintenir notre potentiel militaire ainsi que le sacrifice que doit payer le Peuple, il n'est maintenant plus possible de discuter sur le bien-fondé ou non de l'utilisation des peuples des territoires extérieurs [au sein de l'empire colonial], car cela est devenu au contraire une urgence sur laquelle nous devons nous concentrer ${ }^{18}$, le gouvernement décida le 8 mai 1942 de mettre en œuvre la conscription pour les colonisés à compter de l'année suivante. On peut penser que la menace de la conscription joua également lors des recrutements de gardiens pour les prisonniers de guerre.

La condamnation à mort de Cho Munsang comme auxiliaire militaire de l'armée japonaise, ainsi que celles de vingt-deux autres gardiens coréens dans la même situation correspondent à l'aboutissement final d'un enchaînement de nombreuses couches de violence juridique, qui ont permis la domination coloniale japonaise en Corée ainsi que la mobilisation de ces populations en temps de guerre. La même remarque peut être faite concernant les vingt et un auxiliaires militaires taïwanais condamnés à mort. Tous ces individus qui ont agi en suivant les ordres de leurs supérieurs japonais durant les guerres menées par l'armée japonaise furent jugés en tant que "Japonais" dans le cadre des procès organisés par les armées alliées. Autrement dit, toutes ces personnes se sont vu imputer la responsabilité de guerre du Japon; cette donnée est indissociable du fait que les armées alliées n'ont pas questionné la domination coloniale japonaise. Alors que la Déclaration du Caire ( $1^{\mathrm{er}}$ décembre 1943) - à laquelle se réfère la Déclaration de Potsdam (26 juillet 1945) stipulait que « les trois grandes puissances susmentionnées, ayant conscience de l'état d'esclavage dans lequel se trouve le peuple de Corée, sont déterminées à ce que la Corée devienne libre et indépendante ${ }^{19}$ ", pourquoi les armées alliées n'ont-elles pas soulevé la question de la colonisation japonaise lors des différents procès? Au-delà de la responsabilité du "Généralissime » - le commandant suprême qu'était l'empereur - ou de celle des décideurs japonais civils et militaires, cette absence ne provient-elle pas du fait que soulever le problème de la colonisation aurait empêché de passer en jugement et de condamner à mort tous ces auxiliaires coréens et taïwanais, situés aux extrémités du système militaire japonais? En fait, ce problème a été discuté dans les tribunaux locaux pour les criminels de catégories $\mathrm{B}$ et $\mathrm{C}$. Dans les minutes du procès de $\mathrm{Yi}$ Hangnae 李鶴来 (né en 1925), un autre gardien de camp coréen, condamné à mort comme Cho Munsang, on peut lire l'échange suivant entre le procureur et l'avocat de Yi, l'avocat considérant qu'il n'y avait aucune raison que ce dernier soit inculpé.

L'avocat: "Cette cour de justice doit prêter une attention extrêmement importante à la situation de la Corée avant de passer un citoyen coréen en jugement. Je veux dire que si l'on considère que la Corée est elle-même une victime des agressions japonaises, alors juger un Coréen dans un tribunal pour criminels de guerre reviendra à évacuer le crime constitué par cette agression [contre la Corée]. Évacuer le problème constitué par une agression en particulier constitue, d'après les règlements militaires, un crime équivalent à celui de légitimer une agression. " [...]

Le juge : "J'aimerais entendre l'avis du procureur sur cette objection.»

Le procureur: "Je vais répondre de façon extrêmement simple à cette objection. Le fait est que l'accusé lui-même reconnaît avoir été employé par l'Armée de terre de l'Empire japonais. 
Par conséquent, il a un devoir de fidélité à l'égard de l'Empereur. Cela me semble suffisant pour savoir si nous avons le droit de le passer en jugement. $»^{20}$

L'objection légitime de l'avocat, qui évoque Jacques Vergès lors de ses plaidoyers contre la colonisation lors des procès des membres du FLN à Alger durant la Guerre d'Algérie, fut ainsi sèchement rejetée. De la même façon, lors d'entretiens qui eurent lieu les 11 et 13 décembre 1945 à Singapour entre les autorités britanniques et le procureur général hollandais pour les Indes orientales néerlandaises (actuelle Indonésie), la GrandeBretagne et les Pays-Bas décidèrent d'un commun accord de "traiter les Coréens en tant que Japonais pour ce qui touche aux crimes de guerre $»^{21}$. Interroger la domination coloniale japonaise et les responsabilités afférentes aurait amené à soulever la question de la domination coloniale exercée par les pays alliés eux-mêmes, notamment les ÉtatsUnis, la Grande-Bretagne, les Pays-Bas et la France. Ces deux derniers pays étaient d'ailleurs représentés par des juges dans les procès pour crimes de guerre, alors même qu'ils menaient une guerre contre le mouvement de libération nationale en Indonésie pour le premier, et tout en ayant affirmé la restauration de sa souveraineté sur l'Indochine pour le second. Dès lors, il était impossible que la question coloniale soit soulevée au cours de procès pour crimes de guerre organisés par ces pays. Une violence spécifique du colonialisme, qui traverse le Japon et les pays occidentaux, apparaît de la sorte au sein de la violence légale déployée dans ces procès pour crimes de guerre à l'encontre d'auxiliaires militaires japonais originaires des colonies, tel Cho Munsang.

Malgré tout, le procureur cité plus haut soulignait que Yi Hangnae a «été employé par l'Armée de terre de l'empire japonais », et qu'en conséquence «il a[vait] un devoir de fidélité à l'égard de l'Empereur. » Dans l'armée japonaise, les ordres donnés par les supérieurs devaient être impérativement respectés, et il n'y avait absolument aucune contestation possible de la part des auxiliaires militaires, situés tout en bas de la hiérarchie. Ce devoir d'obéissance absolue s'explique par le fait que les ordres des gradés étaient considérés comme des ordres émanant de l'empereur lui-même, comme cela était spécifié dans l'Admonition aux soldats (Gunjin chokuyu 軍人勅諭, 1882) formulée par l'empereur Meiji, le « Généralissime ", à l'intention des soldats et officiers de «l'armée impériale» (kōgun 皇軍) - qui stipulait : «Quand les soldats d'un rang inférieur reçoivent un ordre d'un supérieur hiérarchique, ils doivent l'interpréter comme un ordre émanant directement de Ma Personne»下級ノモノ八上官ノ命ヨ承 ルコト実ハ直二朕力命ヨ承ル義ナリト心得ヨ. Ce 《devoir de fidélité » que Yi Hangnae avait vis-à-vis de «l'Empereur japonais » correspondait à ce type de relation. Mais dans ce cas, pourquoi est-ce que le commandant suprême, c'est-à-dire l'empereur lui-même qui était responsable des ordres donnés, n'a pas été jugé tandis que tous les auxiliaires militaires qui ont obéi aux ordres des supérieurs hiérarchiques, donc aux ordres de l'empereur, ont, eux, été traduits devant les tribunaux? Les procès des criminels de guerre de catégories B et C organisés par les pays alliés furent non seulement marqués par l'arbitraire dans le choix des prévenus et par le caractère sommaire des procédures suivies, mais ils comportaient ainsi clairement deux contradictions majeures, comme nous l'avons noté ci-dessus. Ces deux contradictions se retrouvent dans le Procès de Tōkyō, qui jugea les criminels de guerre de catégorie $\mathrm{A}$ sans évoquer lui non plus la question coloniale ni la responsabilité de l'empereur. Une immense violence juridique s'est ainsi abattue sans pitié sur les auxiliaires militaires originaires des colonies, tel Cho Munsang, situés aux extrémités de l'armée japonaise. 


\section{Après la mort, vous irez au Yasukuni}

condamnés à mort comme criminels de catégories $\mathrm{B}$ et $\mathrm{C}$ sont commémorés au sein de la célébration commune du Yasukuni, au titre de «martyrs de Shōwa ». Au total, 21181 militaires et auxiliaires militaires coréens, ainsi que 28863 militaires et auxiliaires militaires taïwanais sont célébrés dans ce sanctuaire. La majeure partie de ces personnes sont des Coréens et des Taïwanais mobilisés dans le cadre de la Guerre de l'Asie et du Pacifique (1937-1945), et morts au combat, mais quarante-quatre d'entre eux sont des condamnés à mort pour crimes de guerre. Les noms de ces vingt-trois Coréens sont également gravés sur la «Stèle funéraire pour les martyrs de Shōwa exécutés à mort par décision de justice » du Kōyasan Oku no in, et parmi eux figurent les noms de Hong Sa'ik et de Cho Munsang. Le sanctuaire Yasukuni avait fini d'ajouter l'ensemble des criminels de guerre de catégories B et $\mathrm{C}$ au sein de sa célébration commune dès le mois d'octobre 1966, tandis que les criminels de guerre de catégorie A ont été ajoutés en 1978. On ne peut que s'interroger sur le fait de célébrer ces personnes en ce lieu.

16 Les anciens Premiers ministres de 1941 à 1945 Tōjō Hideki 東條英機 (1884-1948) et Koiso Kuniaki 小磯國昭 (1880-1950) - Koiso fut aussi Gouverneur général de Corée de 1942 à 1944 - figurent parmi les criminels de guerre de catégorie A célébrés au sanctuaire. Si, pour les raisons que nous avons déjà notées, sa responsabilité coloniale ne fut pas soulevée, Koiso est cependant celui qui organisa la conscription en Corée et qui mena l'effort de mobilisation des Coréens au moment de la guerre, après sa nomination comme Gouverneur général en mai 1942. Autrement dit, Cho Munsang et les autres Coréens sont célébrés au Yasukuni en tant que «divinités protectrices du pays » (gokoku no kami 護国の神) avec ceux qui les ont envoyés au champ de bataille, ainsi qu'avec tous les morts qui y sont commémorés de par leurs « hauts exploits » dans la construction et le maintien de l'empire colonial et de la domination sur la Corée, c'est-à-dire aux côtés de leurs agresseurs. La logique du Yasukuni consiste à considérer que Cho Munsang et les autres militaires et auxiliaires militaires originaires des colonies condamnés à mort ont servi de boucs émissaires. Cependant, ce discours qui critique le caractère arbitraire des procès des criminels de guerre organisés par les pays alliés, continue dans un même temps à légitimer la violence juridique qui a finalement conduit Cho Munsang et les autres Coréens en ce lieu et qui légitimait la domination coloniale et la mobilisation dans l'effort de guerre.

Suivant cette logique, Cho Munsang et les autres Coréens continuent encore aujourd'hui, malgré la fin de la domination coloniale japonaise, à être «Japonais ». En février 1979, sept représentants des familles de défunts aborigènes de Taïwan (l'ethnie appelée Takasago 高砂族 avant 1945) sont venus au Japon afin de demander, pour la première fois de la part de familles de défunts des anciennes colonies, à ce que les noms de leurs proches soient retirés de la célébration commune au Yasukuni. Le desservant en second du sanctuaire à l'époque, Ikeda Yoshihachi 池田良八, refusa de retirer ces noms au motif suivant :

Étant donné qu'au moment où ils sont morts au combat ils étaient Japonais, ils ne peuvent pas cesser de l'être après leur mort. Étant donné qu'ils se sont battus comme militaires du Japon, en sachant que s'ils mouraient ils seraient célébrés au Yasukuni, il n'est pas concevable qu'on les en retire à la demande de leur famille. Ces personnes ont demandé à 
collaborer à l'effort de guerre au même titre que les habitants de la métropole, et puisqu'ils ont participé aux combats en tant que Japonais, il est normal de les célébrer au Yasukuni. ${ }^{22}$ l'époque de l'Empire du Grand Japon perdurent au sein du sanctuaire Yasukuni plus d'un demi-siècle après la chute de l'empire colonial et après la fin de la guerre. Le Yasukuni constitue un espace où l'idéologie de l'empire continue d'exister encore de nos jours. Les morts à la guerre originaires des anciennes colonies sont enfermés au milieu de "cette cage qu'est le Yasukuni » ${ }^{24}$, au sein de laquelle ils continuent à être considérés comme "Japonais ». Ainsi, ils continuent d'y être soumis à la domination coloniale, toujours placés sous les ordres de l'empereur. Si le Yasukuni essaie de soustraire les criminels de guerre coréens et taïwanais, tel Cho Munsang, du « jugement injuste » dont ils auraient été les victimes, cela n'est que pour soustraire le Japon luimême du « jugement injuste » dont il serait, selon eux, la victime. Non seulement Cho Munsang et les autres dans son cas se sont vu imputer la responsabilité de guerre du Japon, mais ils continuent à être utilisés par le Japon par-delà leur mort. De façon paradoxale, nous pouvons considérer que les procès des criminels de guerre organisés par les pays alliés et le sanctuaire Yasukuni possèdent des prémisses communes.

Ces prémisses consistent dans le fait de ne pas questionner la domination coloniale japonaise ni la responsabilité de guerre de l'empereur. En conséquence, la seule façon de laver Cho Munsang du «jugement injuste » qui a été prononcé à son encontre, et, dans un même temps, de le libérer de «cette cage qu'est le Yasukuni » et de son état de prisonnier "Japonais ", consiste à poser la question des responsabilités coloniales japonaises et celle de la responsabilité de guerre de l'empereur.

\section{La nationalité japonaise pour tous}

Cho Munsang est aujourd'hui enfermé dans «cette cage qu'est le Yasukuni » avec les autres criminels de guerre de catégories B et $\mathrm{C}$ commémorés. Qu'on nous permette ici de faire l'hypothèse suivante. Si Cho Munsang n'avait pas été condamné à mort, mais avait été relaxé, et que, par conséquent, il n'avait pas été ajouté à la célébration commune du Yasukuni, que ce serait-il passé ? Une telle hypothèse est légitime et justifiée par le fait que d'autres auxiliaires militaires coréens également jugés pour crimes de guerre de catégories B et $\mathrm{C}$, du fait de sévices sur prisonniers de guerre, virent finalement leur peine allégée et furent ensuite relaxés. Ici apparaît une nouvelle fois, avec acuité, l'arbitraire de la violence juridique, qui ne peut être ressentie que comme un « destin », « incertain et ambigu », par ceux qui en furent les jouets. Ce qui a séparé le destin de Cho Munsang de celui de Yi Hangnae, susmentionné, est précisément cet arbitraire.

Yi Hangnae a été condamné à mort par contumace le 20 mars 1947 après à peine deux heures de délibération, pour finalement voir sa peine diminuée à vingt années de prison le 7 novembre de la même année. Il fut ensuite transféré à la prison de Sugamo, à Tōkyō, en août 1951 et bénéficia d'une libération conditionnelle le 6 octobre 1956. Yi

Cipango, 15 | 2008 
servait comme gardien dans un camp de prisonniers à Hintok en Thaïlande, lui aussi aux extrémités du système militaire japonais, et il fut la cible de la colère des prisonniers alliés pour avoir envoyé des prisonniers malades sur les chantiers de construction de la Ligne Thaïlande - Birmanie. D'un autre côté, Yi, qui a décrit dans ses carnets le caractère approximatif des procès des criminels de guerre de catégories $B$ et C, y a noté les sévices dont lui et d'autres furent l'objet de la part des troupes alliées durant leur séjour en prison, attendant leur jugement ou leur exécution ${ }^{25}$.

En août 1951, alors que la péninsule coréenne comportait déjà deux États distincts - la République de Corée au sud, et la République démocratique populaire de Corée, au nord, toutes deux fondées en 1948 - et que de nombreux Coréens qui étaient au Japon durant la guerre étaient retournés chez eux après la défaite, les criminels de guerre de catégories $B$ et $C$ tel Yi Hangnae furent transférés sur le sol japonais où ils n'avaient en réalité jamais mis les pieds. Les pays alliés transférèrent ainsi les auxiliaires militaires coréens au Japon de la même façon qu'ils les avaient jugés, c'est-à-dire " en tant que Japonais ». Parallèlement, les dix-sept personnes inculpées dans le cadre de la seconde partie du Procès de Tōkyō, qui n'eut finalement jamais lieu, et incarcérées à la prison de Sugamo, dont le futur Premier ministre Kishi Nobusuke 岸信介 (1896-1987), furent libérées sans jugement dès le lendemain de l'exécution des sept criminels de guerre de catégorie A exécutés le 23 décembre 1948, dont Tōjō Hideki.

Les contradictions soulevées par l'incarcération des criminels de guerre de catégories B et C tel Yi Hangnae devinrent encore plus saillantes avec l'entrée en vigueur du Traité de paix de San Francisco (signé en 1951) le 28 avril 1952 et avec le retour de la souveraineté japonaise. L'article 11 de ce traité stipule que le gouvernement japonais accepte les verdicts (judgments; jap. hanketsu 判決) des procès des criminels de guerre et devra veiller à l'accomplissement des peines prononcées contre les membres de la «nation japonaise ». Pourtant, le gouvernement japonais ne donna aucune liberté de choisir leur nationalité aux Coréens et aux Taïwanais ; au contraire, il la leur retira de façon unilatérale, comme cela est stipulé dans une directive du 19 avril 1952 (« La Corée et Taïwan seront séparés du territoire japonais à compter du jour d'entrée en vigueur du traité, et en conséquence les Coréens et les Taïwanais, y compris ceux résidant sur le sol métropolitain, perdront tous la nationalité japonaise» 朝鮮および台湾は、条約の 発効の日から、日本国の領土から分離することになるので、これに伴い、朝鮮人 及び台湾人は、内地に在住している者を含めて、全て日本の国籍を喪失する $)^{26}$. Selon cette directive, Yi Hangnae et les autres ne devaient plus faire partie de la «nation japonaise » dès l'entrée en vigueur du Traité de paix. Ceux-ci pensèrent alors, en toute logique, qu'ils seraient libérés, mais ils restèrent incarcérés. Depuis leur prison, sept Coréens dont Yi lancèrent le 14 juin 1952 une demande de procès afin d'obtenir leur libération en s'appuyant sur la Loi sur la protection des personnes (Jinshin hogo hō 人身保護法), mais cette demande fut repoussée par le Tribunal de Grande Instance le 9 juillet de la même année. Le motif invoqué pour maintenir l'exécution de leur peine était qu'ils remplissaient les deux conditions suivantes : «Ils étaient membres de la nation japonaise au moment où ils se sont vus infliger leur peine » et « Ils étaient déjà incarcérés au Japon juste avant l'entrée en vigueur du Traité de paix ». Face à ce verdict, le ministère des Affaires étrangères sud-coréen publia le communiqué suivant.

Les Coréens appelés criminels de guerre ont été jugés comme Japonais au prétexte qu'ils avaient été incorporés dans l'armée japonaise durant la guerre. Cependant, avec la victoire des pays alliés et l'indépendance de la Corée, ceux-ci ont non seulement retrouvé leur 
nationalité coréenne, mais ne sont plus Japonais conformément au traité de paix passé avec

le Japon. Il est donc illégal de les traiter comme des criminels de guerre. ${ }^{27}$ guerre", promulguée deux jours après l'entrée en vigueur du Traité de paix, fut appliquée de façon rétroactive en remontant jusqu'au $1^{\mathrm{er}}$ avril 1952: par conséquent, les criminels de guerre japonais de catégories B et C entraient dans son champ d'application. Yi Hangnae et les autres Coréens qui étaient dans la même prison possédaient la nationalité japonaise jusqu'au 28 avril, et espéraient logiquement bénéficier de cette loi - après tout, ils avaient été déplacés au Japon précisément pour pouvoir y purger leur peine en tant que Japonais. Cependant, ce texte de loi stipulait dans sa « règle annexe $n^{\circ} 2$ » que : « Les personnes n'entrant pas dans le cadre de la Loi sur l'état civil ne se verront pas appliquer la présente loi pour le moment »: ainsi les Coréens et les Taïwanais habitant en métropole, mais ne possédant de fait pas d'état civil japonais (cf. supra) furent exclus de l'application de cette loi. Après leur libération conditionnelle, Yi Hangnae et les autres Coréens ne bénéficièrent d'aucun soutien de la part de l'État japonais, et ne purent pas non plus rentrer en Corée du fait des désordres consécutifs à la Guerre de Corée (1950-1953), souffrant de la pauvreté et de la ségrégation dans un Japon où ils vivaient pour la première fois. Depuis 1956 et jusque récemment, ils ont émis des demandes de réparation auprès des gouvernements japonais successifs, y compris au titre de familles de condamnés à mort, mais ces demandes ne furent jamais acceptées. En 1991, ils déclenchèrent un "procès de demande de réparation à l'État pour les criminels de guerre coréens ", mais cette demande fut repoussée en première et deuxième instances pour finalement échouer du fait d'un rejet par le Tribunal de Grande Instance en décembre 1999.

Faisons le point sur le processus décrit ci-dessus, où se chevauchent impérialisme et colonialisme et qui montre en toute clarté les grands présupposés communs aux puissances occidentales et au Japon. Malgré la chute de l'Empire du Grand Japon et la libération de la Corée, Yi Hangnae et Cho Munsang furent jugés et condamnés à mort « en tant que Japonais ». Cependant, à la différence de Cho, la peine de Yi fut allégée et celui-ci fut transféré au Japon, où il perdit de façon unilatérale sa nationalité japonaise suite à la conclusion du Traité de paix de San Francisco. Mais alors qu'il s'est vu déchu de sa nationalité japonaise, il a quand même dû purger sa peine parce qu'il avait été condamné « en tant que Japonais ». Et pourtant, il fut exclu des indemnisations d'aprèsguerre ou des indemnisations pour les familles des défunts et pour les blessés de guerre, car, en tant que Coréen, ne pouvait s'appliquer pour lui la « Loi sur l'état civil ». Tout au long de ce processus en quatre temps, Yi Hangnae et les autres Coréens condamnés comme criminels de guerre de catégories $\mathrm{B}$ et $\mathrm{C}$ furent les jouets de la violence juridique des pays alliés et du Japon, dans un commun mépris du fait colonial et de la responsabilité de l'empereur, deux données qui à elles seules permettent de comprendre ce qu'était le statut de ces militaires et auxiliaires militaires originaires des colonies, du temps de l'empire colonial. On pourrait ainsi comparer leur destin avec celui des militaires et auxiliaires militaires de l'empire français, notamment les Harkis.

Cho Munsang et Yi Hangnae furent tous deux condamnés à mort lors des procès pour criminels de guerre de catégories $B$ et $C$. Cho fut exécuté et, en conséquence, est aujourd'hui encore célébré au sanctuaire Yasukuni, toujours prisonnier à l'intérieur du «Japon ». Inversement, Yi Hangnae a pu échapper à l'exécution de sa peine, mais pour aussitôt être déchu de sa nationalité japonaise et se voir refuser de façon continue tout soutien ou indemnité. Certes, il a pu ainsi éviter d'être célébré au Yasukuni, mais on

Cipango, 15 | 2008 
peut considérer que Yi ne fut jamais réellement libéré après la guerre et après la décolonisation. Dans un premier temps, il fit l'objet d'une politique d'assimilation à outrance au sein de la nation japonaise, pour, dans un second temps, s'en voir totalement exclu. Cette contradiction illustre parfaitement la nature de la violence étatique appuyée sur la Loi, qui constitue une dimension constitutive des États et que l'on doit prendre en considération pour réfléchir sur les relations entre la métropole et ses colonies à l'époque des empires coloniaux, ainsi que, pour après la chute des empires coloniaux, à propos de la situation ambiguë de ces personnes qui furent particulièrement liées à l'ancienne métropole.

\section{NOTES}

1. Sur le sanctuaire Yasukuni, voir mon ouvrage: Yasukuni mondai 靖国問題, Tōkyō, Chikuma shobō 筑摩書房, 2005, traduction française par A. Nanta, Les Belles Lettres, 2012.

2. D’après la brochure officielle actuellement distribuée par le sanctuaire : Yasukuni daihyakka や すくに大百科 (Encyclopédie du Yasukuni), Yasukuni jinja shamukyoku.

3. Chongqing était la capitale de la Chine nationaliste et du gouvernement de Jiang Jièshi (Tchang Kaï-chek) après la chute de Nankin en décembre 1937. [NDT.]

4. Données provenant des archives du ministère de la Justice japonais.

5. Cité dans l'enquête menée par la chaîne NHK «Cho Munsang no isho »チョウ・ムンサンの遺 書 (Le testament de Cho Munsang), diffusée le 15 août 1991.

6. Walter Benjamin, «Critique de la violence », 1921, in Euvres, t. 1, Paris, Folio, 2000, p. 210-243. Idem pour les citations qui suivent.

7. Propos du procureur John Williams, voir note 5.

8. W. Benjamin, op. cit.

9. Dans le monde sinisé, le patronyme précède le nom personnel.

10. Sur ce point, voir: Hayashi Hirofumi 林博史, BC kyū senpan saiban B C級戦犯裁判 (Les procès des criminels de guerre de catégories B et C), Tōkyō, Iwanami shoten 岩波書店, 2005.

11. En actuelle République démocratique populaire de Corée, Kaesòng était la capitale du royaume unifié de Koryò (912-1392). [NDT.]

12. Cité à partir de l'enquête NHK, voir note 5 .

13. Kaneko était le nom japonais d'un autre Coréen condamné à mort, dont le nom coréen était Kim Changnok 金長録.

14. Cité d'après: Sugamo isho hensan kai 巣鴨遺書編纂会 (Société pour la compilation des testaments de la prison de Sugamo), Seiki no isho 世紀の遺書 (Les testaments du $\mathrm{xx}^{\mathrm{e}}$ siècle), Tōkyō, Mainichi shinbun-sha 毎日新聞社, 1953.

15. On traduit le mot japonais kōminka 皇民化 par le néologisme de Hannah Arendt, qu'elle utilise dans cette acception relativement à l'assimilation des populations colonisées (Les origines du totalitarisme, t. 2., L'impérialisme). [NDT.]

16. W. Benjamin, op. cit.

17. NDT. La Kempeitai 憲兵隊 est une force militaire chargée du maintien de l'ordre dans les zones situées hors de la juridiction de la police. De par son statut et sa fonction, elle est grosso modo l'équivalent des forces de gendarmerie en France, qui d'ailleurs avaient la charge du 
maintien de l'ordre et de la répression des rebelles en Algérie française - sauf incident majeur qui voyait l'intervention de l'armée proprement dire, en Algérie comme en Corée. [NDT.]

18. Rikugunshō heibika 陸軍省兵備課 (ministère de l'Armée de terre, section Préparation des soldats), Daitōa sensō ni tomonau waga jinteki kokuryoku no kentō 大東亜戦争二伴つ我力人的国力 ノ検討 (Étude sur le potentiel humain de notre pays dans le cadre de la Guerre de la grande Asie Orientale), 1942.

19. Le texte original en anglais est: "The aforesaid three great powers, mindful of the enslavement of the people of Korea, are determined that in due course Korea shall become free and independent », et en japonais: 《三大国八朝鮮ノ人民ノ奴隷状態二留意シ㗹テ朝鮮 且独立ノモノタラシムルノ決意习有ス». [NDT.]

20. Sakurai Hitoshi 桜井均, Terebi wa sensōo o dō egaite kita ka テレビは戦争をどう描いてきたか (Comment la télévision a-t-elle présenté la guerre ?), Tōkyō, Iwanami shoten, 2005, p. 374. Souligné par l'auteur.

21. Utsumi Aiko 内海愛子, Chōsenjin BC kyū senpan no kiroku 朝鮮人 $B$ C 級戦犯の記録 (Les minutes des procès des criminels de guerre coréens de catégories B et C), Tōkyō, Keisō shobō 勁 草書房, 1982, p. 159.

22. Asahi shinbun, 16 avril 1987.

23. Tsunoda Saburō 角田三郎, Yasukuni to chinkon 靖国と鎮魂 (Le Yasukuni et la commémoration aux morts), Tōkyō, San.ichi shobō 三一書房, 1977, p. 266.

24. Selon la formulation de Sugawara Ryūken. Sugawara Ryūken 菅原瀧憲, “Yasukuni » to iu ori kara no kaihō「靖国」という檻からの解放 (Etre libéré de cette cage qu'est le Yasukuni), Tōkyō, Nagada Bunshō-dō 永田文昌堂, 2005.

25. Yi Hangnae (Ri Kakurai) 李鶴来, Changi miketsu kōryū no taiken チャンギ未決拘留の体娩 (Mon expérience d'emprisonnement sans conclusion à Changi), in Utsumi Aiko 内海愛子, Gavan McCormack G・マコーマック, Hank Nelson H・ネルソン (dir.), Taimen tetsudō to Nihon no sensō sekinin 泰緬鉄道と日本の戦争責任 (La Ligne Thailande - Birmanie et la responsabilité de guerre japonaise), Ōsaka, Akashi shoten 明石書店, 1994, p. 307 et suiv.

26. Hōmufu minjikyokuchô tsūtatsu 法務府民事局長通達, daté du 19 avril 1952. [NDT.]

27. Cité d'après le Mainichi shinbun, 11 septembre 1952.

\section{RÉSUMÉS}

Après la guerre, les procès des criminels de catégories $B$ et $C$ originaires des colonies furent marqués par l'arbitraire et le caractère sommaire des procédures.

After the war, Class B/C War Criminals also included ethnic Korean and Taiwanese. Their trials were marked by summary justice. 
INDEX

\section{Index géographique : Corée}

キーワード : sensō hanzai 戦争犯罪, sensō hanzainin 戦争犯罪人, kioku 記憶, kankoku hei 韓国 兵, Yasukuni jinja 靖国神社, Kankoku 韓国, Taiheiyō sensō 太平洋戦争 (1941-1945), Tōkyō saiban 東京裁判 (1946-1948), shigakushi 史学史, rekishi 歴史

Index chronologique : guerre du Pacifique (1941-1945), Tōkyō - procès de (1946-1948)

Keywords : Historiography, History, Korea, Memory, Orientalism, War of the Pacific (1939-1945), Tōkyō trial (1946-1948), War Criminals, Yasukuni mondai

Mots-clés : crimes de guerre, criminels de guerre, mémoire, militaires coréens, sanctuaire Yasukuni 